Cochrane Database of Systematic Reviews

\title{
Probing for congenital nasolacrimal duct obstruction (Review)
}

Petris C, Liu D

Petris C, Liu D.

Probing for congenital nasolacrimal duct obstruction.

Cochrane Database of Systematic Reviews 2017, Issue 7. Art. No.: CD011109.

DOI: 10.1002/14651858.CD011109.pub2.

www.cochranelibrary.com 
TABLE OF CONTENTS

ABSTRACT

PLAIN LANGUAGE SUMMARY

SUMMARY OF FINDINGS

BACKGROUND

OBJECTIVES

METHODS

RESULTS

Figure 1.

Figure 2.

DISCUSSION

AUTHORS' CONCLUSIONS

ACKNOWLEDGEMENTS

REFERENCES

CHARACTERISTICS OF STUDIES

DATA AND ANALYSES

Analysis 1.1. Comparison $1 \mathrm{Immediate}$ probing vs observation/deferred probing, Outcome 1 Treatment success at 18 months of age.

APPENDICES

CONTRIBUTIONS OF AUTHORS

DECLARATIONS OF INTEREST

SOURCES OF SUPPORT

DIFFERENCES BETWEEN PROTOCOL AND REVIEW

INDEX TERMS 
[Intervention Review]

\section{Probing for congenital nasolacrimal duct obstruction}

Carisa Petris ${ }^{1}$, Don Liu ${ }^{2}$

1Ophthalmic Plastic, Reconstructive, and Orbital Surgery, Mason Eye Institute, University of Missouri Health Care, Columbia, Missouri, USA. ${ }^{2}$ Department of Ophthalmology, Mason Eye Institute, University of Missouri Health Care, Columbia, Missouri, USA

Contact: Don Liu, Department of Ophthalmology, Mason Eye Institute, University of Missouri Health Care, Columbia, Missouri, 65211, USA. liud@health.missouri.edu.

Editorial group: Cochrane Eyes and Vision Group.

Publication status and date: New, published in Issue 7, 2017.

Citation: Petris C, Liu D. Probing for congenital nasolacrimal duct obstruction. Cochrane Database of Systematic Reviews 2017 , Issue 7. Art. No.: CD011109. DOI: 10.1002/14651858.CD011109.pub2.

Copyright @ 2017 The Cochrane Collaboration. Published by John Wiley \& Sons, Ltd.

\section{A B S T R A C T}

\section{Background}

Congenital nasolacrimal duct obstruction (NLDO) is a common condition causing excessive tearing in the first year of life. Infants present with excessive tearing or mucoid discharge from the eyes due to blockage of the nasolacrimal duct system, which can result in maceration of the skin of the eyelids and local infections, such as conjunctivitis, that may require antibiotics. The incidence of nasolacrimal duct obstruction in early childhood ranges from $5 \%$ to $20 \%$ and often resolves without surgery. Treatment options for this condition are either conservative therapy, including observation (or deferred probing), massage of the lacrimal sac and antibiotics, or probing the nasolacrimal duct to open the membranous obstruction at the distal nasolacrimal duct. Probing may be performed without anesthesia in the office setting or under general anesthesia in the operating room. Probing may serve to resolve the symptoms by opening the membranous obstruction; however, it may not be successful if the obstruction is due to a bony protrusion of the inferior turbinate into the nasolacrimal duct or when the duct is edematous (swollen) due to infection such as dacryocystitis. Additionally, potential complications with probing include creation of a false passage and injury to the nasolacrimal duct, canaliculi and puncta, bleeding, laryngospasm, or aspiration.

\section{Objectives}

To assess the effects of probing for congenital nasolacrimal duct obstruction.

\section{Search methods}

We searched the Cochrane Central Register of Controlled Trials (CENTRAL), which contains the Cochrane Eyes and Vision Trials Register (2016, Issue 8); MEDLINE Ovid (1946 to 30 August 2016); Embase.com (1947 to 30 August 2016); PubMed (1948 to 30 August 2016); LILACS (Latin American and Caribbean Health Sciences Literature Database; 1982 to 30 August 2016), the metaRegister of Controlled Trials ( $m$ RCT) (www.controlled-trials.com), last searched 14 August 2014; ClinicalTrials.gov (www.clinicaltrials.gov), searched 30 August 2016; and the WHO International Clinical Trials Registry Platform (ICTRP) (www.who.int/ictrp/search/en), searched 30 August 2016. We did not use any date or language restrictions in the electronic searches for trials.

\section{Selection criteria}

We included randomized controlled trials (RCTs) that compared probing (office-based or hospital-based under general anesthesia) versus no (or deferred) probing or other interventions (observation alone, antibiotic drops only, or antibiotic drops plus massage of the nasolacrimal duct). We did not include studies that compared different probing techniques or probing compared with other surgical procedures. We included studies in children aged three weeks to four years who may have presented with tearing and conjunctivitis.

\section{Data collection and analysis}

Two review authors independently screened studies for inclusion and independently extracted data and assessed risk of bias for the included studies. We analyzed data using Review Manager software and evaluated the certainty of the evidence using GRADE. 


\section{Main results}

We identified two RCTs and no ongoing studies; one of the included RCTs was registered. The studies reported on 303 eyes of 242 participants who had unilateral or bilateral congenital nasolacrimal duct obstruction. For both included studies, the interventions compared were immediate office-based probing to remove the duct obstruction versus deferred probing, if needed, after 6 months of observation or once the child reached a certain age.

The primary outcome of the review, treatment success at 6 months, was reported partially in one study. Treatment success was not reported at this time point for all children in the immediate probing group; however, 77 of 117 (66\%) eyes randomized to deferred probing had resolved without surgery 6 months after randomization and 40 (34\%) eyes did not resolve without probing. For children who had unilateral NLDO, those randomized to immediate probing had treatment success more often than those who were randomized to deferred probing (RR $1.41,95 \% \mathrm{Cl} 1.12$ to $1.78 ; 163$ children; moderate-certainty evidence). Treatment success for all children was assessed in the study at age 18 months; as an ad hoc analysis in the included study, results were presented separately for children with unilateral and bilateral NLDO (RR $1.13,95 \% \mathrm{Cl} 0.99$ to 1.28 and RR $0.86,95 \% \mathrm{Cl} 0.70$ to 1.06 , respectively; very low-certainty evidence).

In the other small study (26 eyes of 22 children), more eyes that received immediate probing were cured within one month after surgery compared with eyes that were randomized to deferred probing and analyzed at age 15 months (RR $2.56,95 \% \mathrm{Cl} 1.16$ to 5.64 ). We considered the evidence to be low-certainty due to imprecision from the small study size and risk of bias concerns due to attrition bias.

One study reported on the number of children that required reoperation; however, these data were reported only for immediate probing group. Nine percent of children with unilateral NLDO and 13\% with bilateral NLDO required secondary procedures.

One study reported cost-effectiveness of immediate probing versus deferred probing. The mean cost of treatment for immediate probing was less than for deferred probing; however, there is uncertainty as to whether there is a true cost difference (mean difference USD -139, 95\% CI USD - 377 to 94 ; moderate-certainty evidence).

Reported complications of the treatment were not serious. One study reported that there were no complications for any surgery and no serious adverse events, while the other study reported that bleeding from the punctum occurred in $20 \%$ of all probings.

\section{Authors' conclusions}

The effects and costs of immediate versus deferred probing for NLDO are uncertain. Children who have unilateral NLDO may have better success from immediate office probing, though few children have participated in these trials, and investigators examined outcomes at disparate time points. Determining whether to perform the procedure and its optimal timing will require additional studies with greater power and larger, well-run clinical trials to help our understanding of the comparison.

\section{PLAIN LANGUAGE SUMMARY}

\section{Using a probe to clear a blockage in a child's tear duct}

\section{What is the aim of this review?}

Sometimes children are born with a blocked tear duct. The aim of this Cochrane Review was to find out whether it is better to immediately clear this blocked tear duct, using a probe, or to wait to see if the blockage clears on it's own. Cochrane review authors collected and analyzed all relevant studies to answer this question and found two studies.

\section{Key messages}

It is not clear whether immediate probing results in more treatment success than waiting for the blockage to clear on its own. In children with only one eye affected, immediate probing may be more beneficial than waiting.

\section{What was studied in this review?}

In normal newborn eyes, the tear or nasolacrimal duct allows for drainage of tears. Some babies are born with a blockage in the nasolacrimal duct that creates excessive tearing. This condition is known as congenital nasolacrimal duct obstruction (NLDO). Although the condition often resolves on its own, children with NLDO have a greater chance of infections in the eye and eyelid. Treatment options for NLDO include regular observation to determine whether the condition resolves on its own, massaging the tear duct (the inner corner of the eye, by the nose), or probing, which involves inserting a probe into the duct to relieve the blockage. Probing is a minor procedure that can be performed with or without anesthesia. While probing may resolve symptoms of NLDO, there are potential complications. The aim of this review is to assess the safety and success of probing to treat congenital NLDO and see if it results in better treatment success than waiting to see if the blockage clears on its own.

\section{What are the main results of the review?}

This review included two studies including 303 eyes in 242 children. Both studies compared immediate probing versus regular observation to see if the blockage resolved on its own. The first study looked at children with blockage in one or both eyes. In children with only one affected eye, immediate probing was more successful in treating NLDO at six months. In children with blockage in both eyes, it was unclear whether immediate probing was more effective than observation (and delayed probing if the blockage did not resolve on its own). The second study found that children who received probing immediately were cured by one to three months after the procedure more often 
than the children who had observation and waited to probe. This may indicate that immediate probing is a better option than waiting for babies with NLDO.

This review included two studies that were conducted differently, so it's not definitive whether probing is more successful than waiting. There did not appear to be an inherent risk in using probing to treat NLDO, however the studies were small and may not have identified potentially rare side effects. The cost of probing might be less, but it depends on whether the baby needs antibiotics after treatment. More studies are needed to assess the comparative safety and effectiveness of probing as a treatment option for children with congenital NLDO.

How up-to-date is this review?

Cochrane review authors searched for studies that had been published up to 30 August 2016. 
SUMMARY OF FINDINGS

\section{Summary of findings for the main comparison.}

Immediate office-based probing compared with deferred probing for congenital nasolacrimal duct obstruction

Patient or population: children with congenital nasolacrimal duct obstruction (NLDO)

Intervention: immediate office-based probing

Comparison: deferred probing, if needed, after an observation period

\begin{tabular}{|c|c|c|c|c|c|c|}
\hline \multirow[t]{3}{*}{ Outcomes } & \multicolumn{2}{|c|}{$\begin{array}{l}\text { Illustrative comparative risks }{ }^{\star} \\
(95 \% \mathrm{Cl})\end{array}$} & \multirow[t]{3}{*}{$\begin{array}{l}\text { Relative effect } \\
(95 \% \mathrm{CI})\end{array}$} & \multirow{3}{*}{$\begin{array}{l}\text { No of partici- } \\
\text { pants } \\
\text { (studies) }\end{array}$} & \multirow{3}{*}{$\begin{array}{l}\text { Certainty of } \\
\text { the evidence } \\
\text { (GRADE) }\end{array}$} & \multirow[t]{3}{*}{ Comments } \\
\hline & Assumed risk & $\begin{array}{l}\text { Corresponding } \\
\text { risk }\end{array}$ & & & & \\
\hline & $\begin{array}{l}\text { Deferred prob- } \\
\text { ing if needed }\end{array}$ & $\begin{array}{l}\text { Immediate of- } \\
\text { fice-based prob- } \\
\text { ing }\end{array}$ & & & & \\
\hline $\begin{array}{l}\text { Treatment suc- } \\
\text { cess } \\
\text { (at } 6 \text { months) }\end{array}$ & See comment & & - & - & $\begin{array}{l}\oplus \oplus \oplus \ominus \\
\text { Moderate } a\end{array}$ & $\begin{array}{l}\text { In one study, among participants with unilateral NL- } \\
\text { DO, treatment success at } 6 \text { months occurred more of- } \\
\text { ten in the immediate probing group compared with } \\
\text { the deferred probing group (RR } 1.41,95 \% \mathrm{Cl} 1.12 \text { to } \\
1.78 ; 163 \text { participants). Data for participants with bilat- } \\
\text { eral NLDO were not fully reported in this study. }\end{array}$ \\
\hline \multirow{5}{*}{$\begin{array}{l}\text { Treatment suc- } \\
\text { cess } \\
\text { (at other time } \\
\text { points) }\end{array}$} & \multicolumn{2}{|c|}{$\begin{array}{l}\text { Treatment success at } 18 \text { months of } \\
\text { age in participants with unilateral } \\
\text { NLDO }\end{array}$} & \multirow[t]{2}{*}{$\begin{array}{l}\text { RR } 1.13 \text { (0.99 to } \\
1.28 \text { ) }\end{array}$} & \multirow[t]{2}{*}{$\begin{array}{l}146 \\
(1 \mathrm{RCT})\end{array}$} & \multirow[t]{4}{*}{$\begin{array}{l}\oplus \odot \odot \odot \\
\text { Very low a,b,c }\end{array}$} & \multirow{4}{*}{$\begin{array}{l}\text { Two studies reported on treatment success at oth- } \\
\text { er time points, but the data could not be combined } \\
\text { as the studies used different definitions of treatment } \\
\text { success and measured the outcome at different time } \\
\text { points. } \\
\text { One study reported treatment success when partic- } \\
\text { ipants were } 18 \text { months old, which ranged from } 9-12 \\
\text { months after randomization, depending on the age of } \\
\text { the child at enrollment. The outcome was defined as } \\
\text { the absence of clinical signs of NLDO. The data was re- } \\
\text { ported in subgroups for participants with unilateral } \\
\text { and bilateral NLDO. }\end{array}$} \\
\hline & 817 per 1000 & $\begin{array}{l}\mathbf{9 2 3} \text { per } \mathbf{1 0 0 0} \\
\text { (809 to } 1000)\end{array}$ & & & & \\
\hline & \multicolumn{2}{|c|}{$\begin{array}{l}\text { Treatment success at } 18 \text { months of } \\
\text { age in participants with bilateral } \\
\text { NLDO }\end{array}$} & \multirow[t]{2}{*}{$\begin{array}{l}\text { RR } 0.86 \text { ( } 0.70 \text { to } \\
1.06 \text { ) }\end{array}$} & \multirow[t]{2}{*}{$\begin{array}{l}108 \\
(1 \mathrm{RCT})\end{array}$} & & \\
\hline & 820 per 1000 & $\begin{array}{l}705 \text { per } 1000 \\
\text { (574 to } 869)\end{array}$ & & & & \\
\hline & \multicolumn{2}{|c|}{$\begin{array}{l}\text { Cure (including a normal FDDT) at } \\
\text { one month for participants who }\end{array}$} & $\begin{array}{l}\text { RR } 2.56 \text { (1.16 to } \\
5.64)\end{array}$ & $\begin{array}{l}26 \\
(1 \mathrm{RCT})\end{array}$ & $\begin{array}{l}\oplus \oplus \ominus \ominus \\
\text { Low d,e }\end{array}$ & $\begin{array}{l}\text { 'cure' (complete or near complete remission of symp- } \\
\text { toms and signs including a normal FDDT) and ana- }\end{array}$ \\
\hline
\end{tabular}


had immediate probing and at age

15 months for those who had de-

ferred probing

313 per 1000

800 per 1000

$(363$ to 1,000$)$

\begin{tabular}{|c|c|c|c|c|c|c|}
\hline $\begin{array}{l}\text { Proportion of } \\
\text { participants } \\
\text { requiring sec- } \\
\text { ondary proce- } \\
\text { dures }\end{array}$ & See comment & & - & - & - & $\begin{array}{l}\text { One study reported on the number of children that re- } \\
\text { quired reoperation at a later time point after receiv- } \\
\text { ing immediate office probing. } 9 \% \text { of participants with } \\
\text { unilateral NLDO and } 13 \% \text { with bilateral NLDO required } \\
\text { secondary procedures. The need for secondary proce- } \\
\text { dures was not reported in the participants randomized } \\
\text { to deferred surgery. }\end{array}$ \\
\hline $\begin{array}{l}\text { Cost-effective- } \\
\text { ness }\end{array}$ & $\begin{array}{l}\text { The mean cost } \\
\text { of observa- } \\
\text { tion/deferred } \\
\text { probing was } \\
\text { USD } 701\end{array}$ & $\begin{array}{l}\text { The mean cost } \\
\text { of immediate of- } \\
\text { fice-based prob- } \\
\text { ing was USD } 139 \\
\text { lower } \\
\text { (USD } 377 \text { lower } \\
\text { to USD } 94 \text { higher) }\end{array}$ & - & $\begin{array}{l}163 \\
(1 \mathrm{RCT})\end{array}$ & $\begin{array}{l}\oplus \oplus \oplus \ominus \\
\text { Moderate } f\end{array}$ & - \\
\hline Complications & See comment & & - & - & - & $\begin{array}{l}\text { One study reported that there were no complications } \\
\text { for any surgery, while the other reported that bleed- } \\
\text { ing from the punctum occurred in } 20 \% \text { of all probings } \\
\text { (this number also included participants who were part } \\
\text { of the non-randomized study reported in this publica- } \\
\text { tion). }\end{array}$ \\
\hline
\end{tabular}

*The basis for the assumed risk is the mean control group risk. The corresponding risk (and its 95\% confidence interval) is based on the assumed risk in the comparison group and the relative effect of the intervention (and its $95 \% \mathrm{Cl}$ ).

Cl: confidence interval; RR: risk ratio; FDDT: fluorescein dye disappearance test.

GRADE Working Group grades of evidence

High-certainty: Further research is very unlikely to change our confidence in the estimate of effect.

Moderate-certainty: Further research is likely to have an important impact on our confidence in the estimate of effect and may change the estimate.

Low-certainty: Further research is very likely to have an important impact on our confidence in the estimate of effect and is likely to change the estimate.

Very low-certainty: We are very uncertain about the estimate.

aDowngraded for risk of bias $(-1)$ as the study was judged to have high risk of attrition bias $(12 \%$ in the immediate probing group and $10 \%$ in the deferred probing group did not attend the 18-month primary outcome examination).

b Downgraded for inconsistency $(-1)$ as the effect estimates from different subgroups favored opposing interventions.

c Downgraded for imprecision (-1) as the ad hoc subgroup analysis of data led to imprecision because the study was not powered to examine an effect when participants were v for intervention of immediate probing and at age 15 months for deferred probing). yzed this outcome at different time points depending on group allocation (analyzed within one month 
Downgraded for imprecision (-1) as the RCT portion of the study only included 26 participants.

eDowngraded for risk of bias (-1) as the study was judged to have high risk of attrition bias due to different follow-up times in the intervention and observation group.

fDowngraded for reporting bias (-1) as the other study in this review did not report on this outcome. 


\section{B A C K G R O U N D}

\section{Description of the condition}

Congenital nasolacrimal duct obstruction is a common condition that results in epiphora (excessive tearing) early in life. The lacrimal excretory system is responsible for draining excess tears and is composed of the canaliculi, the lacrimal sac, and the nasolacrimal duct. During development, the nasolacrimal duct originates at the inferomedial aspect of the orbit and grows toward the nose and eye, opening under the inferior turbinate in the nose and on the medial aspect of the upper and lower eyelids. Obstruction in this drainage system at birth is most often due to a membrane at the end of the nasolacrimal duct called the valve of Hasner. Other developmental abnormalities of the lacrimal drainage system may occur, resulting in obstruction of the system, including stenosis (narrowing) of the nasolacrimal duct, canaliculi or puncta, atresia (absence) of the puncta or canaliculus, and rarely, maldevelopment of the entire lacrimal drainage system. A dacryocystocele is a specific type of blockage that occurs when there is both obstruction of the distal nasolacrimal duct and proximal kinking of the common canaliculus prior to the lacrimal sac, resulting in accumulation of secretions within the lacrimal sac.

Nasolacrimal duct obstruction may affect one or both eyes. Infants present with excessive tearing or mucoid discharge from the eyes because the natural drainage system is blocked. Tearing does not always suggest nasolacrimal duct obstruction, as this symptom may occur in other conditions, such as exposure to irritants, eyelid malposition, abnormal eye lashes, or congenital glaucoma, which may be identified on careful examination. Excessive tearing, discharge or both can result in maceration of the skin of the eyelids and conjunctivitis requiring topical antibiotics. Infants with dacryocystocele are also at risk of secondary infection of the lacrimal sac, called dacryocystitis, which may require prompt treatment with intravenous antibiotics and probing of the system (Cassady 1948).

The various estimates of the incidence of nasolacrimal duct obstruction in early childhood reported by different groups of investigators range from 5\% to 20\% (Ffookes 1962; Guerry 1948; MacEwen 1991; Sevel 1981). An eloquent developmental anatomy study by Cassady 1952 found that $73 \%$ of full-term stillborn infants had a membranous obstruction of the distal nasolacrimal duct on histological examination. This study evaluated 15 stillborn infants and proposed that these early nasolacrimal duct obstructions may be undetected clinically, as the membranous obstruction usually opens within the first few weeks of life, prior to the commencement of lacrimation (production of tears) (Cassady 1952; Shekunov 2011). A large, prospective cohort study by MacEwen and Young examined the incidence and natural history of congenital nasolacrimal duct obstruction in the first year of life (MacEwen 1991). In this study, investigators examined a total of 4792 Scottish children born in 1988 at eight weeks of life, evaluating them for signs of congenital nasolacrimal duct obstruction and following them over their first year to monitor symptom resolution. Infants with signs of tearing underwent evaluation in the eye clinic to confirm nasolacrimal duct obstruction with the dye disappearance test. The study found that $20 \%$ of all of the infants had an obstruction in the nasolacrimal duct system and $96 \%$ of the obstructions resolved by one year of age without treatment (MacEwen 1991).

\section{Description of the intervention}

Conservative therapy for this condition includes observation, massage of the lacrimal sac and antibiotics. Probing of the nasolacrimal duct involves passing a probe into the lacrimal sac and down through the nasolacrimal duct to open the obstruction at the lower end of the duct. Physicians can perform the procedure without anesthesia in the office setting or under general anesthesia in the operating room.

\section{How the intervention might work}

Complete resolution of symptoms may result for membranous obstructions that break with the probe. However, probing may not be successful when the obstruction is due to a bony protrusion of the inferior turbinate into the nasolacrimal duct or when the duct is edematous (swollen) due to inflammation (dacryocystitis) (Ffookes 1962; Wesley 1985). Potential complications with probing include creation of a false passage and injury to the nasolacrimal duct, canaliculi and puncta.

\section{Why it is important to do this review}

Although probing is considered an efficacious intervention for congenital nasolacrimal duct obstruction, we have found no systematic review of the existing literature to support this clinical practice. In addition, controversy exists regarding the timing of probing (PEDIG 2012; Tan 2001). The decision to probe early (< 12 months) versus late (> 12 months) is usually based on the surgeons' clinical judgement and experience. Waiting to probe until after one year of age allows for spontaneous resolution of the nasolacrimal duct obstruction and potential avoidance of the procedure altogether. It is also technically easier to perform probing under general anesthesia than in-office probing with restraints. Some studies have shown that late probing has a higher failure rate than early probing (Nelson 1985; Paul 1985; Paul 1994; Petersen 1978; Stager 1992). Many of these studies lacked control groups and were at high risk of selection bias, as many of the children in the early probing group would have resolved without treatment; a high proportion of children who undergo late probing typically have more complicated obstructions that would not have resolved naturally (Paul 1994). Some clinicians advocate early probing to reduce exposure to symptomatic tearing, incur less overall cost and avoid general anesthesia (Schnall 2013; Stager 1992). We aimed to systematically review and synthesize the evidence on probing for congenital nasolacrimal duct obstruction in order to estimate the effectiveness and to compare the effects of early versus deferred nasolacrimal duct probing.

\section{O B JECT IVES}

The primary objective of this systematic review was to assess the effectiveness and safety of probing for congenital nasolacrimal duct obstruction.

\section{METHODS}

\section{Criteria for considering studies for this review}

\section{Types of studies}

We included only randomized controlled trials (RCTs) in this review. We planned to include quasi- and non-randomized trials if we did not find any RCTs, but this was not necessary as we identified RCTs in our searches. 


\section{Types of participants}

We included studies in children with congenital nasolacrimal duct obstruction aged three weeks to four years who may have presented with tearing and conjunctivitis. We included studies with children up to four years of age to allow for outcomes after early versus late probing. We planned to exclude studies with children older than four years because they are more likely to have acquired nasolacrimal duct obstruction. We also excluded studies of children with previous nasolacrimal duct obstruction in order to assess outcomes after primary probing rather than outcomes among failures of initial probing.

We did not include studies in infants with congenital dacryocystocele (distention of the lacrimal sac as a result of a distal nasolacrimal duct obstruction), as these children may develop infection or obstruction of nasal breathing with an inability to feed, and they often require treatment in the first two weeks of life. We also excluded studies that enrolled children with dacryocystitis (infection of the lacrimal sac), keratitis (infection of the cornea), blepharitis (inflammation of the eyelid), cellulitis (infection the skin of the eyelids), trichiasis (lashes that rub on the eye), tumors causing direct compression of the lacrimal drainage system, paralysis of the muscles around the eyelids, eyelid trauma, entropion (inward turning of the eyelid), ectropion (outward turning of the eyelid), epiblepharon (inward turning of the eyelid margin, coloboma (notching or loss of eyelid margin), or other eyelid malposition.

\section{Types of interventions}

We included studies that compared probing (office-based or hospital-based under general anesthesia) versus no (or deferred) probing or other interventions, including observation alone, antibiotic drops only, or antibiotic drops plus massage of the nasolacrimal duct. In this review we did not include studies that compared different probing techniques or probing compared with other surgical procedures.

\section{Types of outcome measures}

\section{Primary outcomes}

The primary outcome for this review was the proportion of participants with treatment success, defined as the absence of clinical signs and symptoms, including epiphora and mucous discharge, at six months after probing.

\section{Secondary outcomes}

Secondary outcomes for this review includes:

1. proportion of participants with treatment success at time points other than six months after probing as data are available (e.g. one year after probing);

2. proportion of participants requiring secondary procedures, surgical or diagnostic, performed within one month, three months, six months, and one year as a consequence of probing or not probing;

3. cost-effectiveness based on estimates through six months;

4. proportion of participants with complications including bleeding, injury to the nasolacrimal system or the eye, or canalicular stenosis (narrowing of the nasolacrimal duct due to fibrosis), other complications of probing and any other complications reported from included trials within six months of probing.

\section{Search methods for identification of studies}

\section{Electronic searches}

The Cochrane Eyes and Vision Information Specialist conducted systematic searches in the following databases for randomized controlled trials and controlled clinical trials. There were no language or publication year restrictions. The date of the search was 30 August 2016.

- Cochrane Central Register of Controlled Trials (CENTRAL; 2016, Issue 8), which contains the Cochrane Eyes and Vision Trials Register, in the Cochrane Library (searched 30 August 2016) (Appendix 1).

- MEDLINE Ovid (1946 to 30 August 2016) (Appendix 2).

- Embase.com (1947 to 30 August 2016) (Appendix 3).

- PubMed (1948 to 30 August 2016) (Appendix 4).

- LILACS (Latin American and Caribbean Health Science Information database (1982 to 30 August 2016) (Appendix 5).

- metaRegister of Controlled Trials (mRCT) (www.controlledtrials.com; last searched 14 August 2014) (Appendix 6).

- US National Institutes of Health Ongoing Trials Register ClinicalTrials.gov (www.clinicaltrials.gov; searched 30 August 2016) (Appendix 7).

- World Health Organization International Clinical Trials Registry Platform (www.who.int/ictrp; searched 30 August 2016) (Appendix 8).

\section{Searching other resources}

We used Scopus to search for publications that cited included studies. We searched the reference lists for studies included in the review to identify any additional ones cited. We did not specifically handsearch journals or conference proceedings for this review.

\section{Data collection and analysis}

\section{Selection of studies}

The two authors independently screened titles and abstracts of trials identified by the electronic searches. We obtained full-text reports of studies that at least one author had classified as definitely or possibly meeting the inclusion criteria for this review. Both authors independently determined from reading the fulltext reports whether each study was eligible for inclusion in the review. We resolved discrepancies through discussion. We provided a description of those studies excluded after full-text review in the 'Characteristics of excluded studies' table. We planned to contact study authors for additional information whenever study eligibility could not be determined from study reports; however, this step was not necessary.

\section{Data extraction and management}

For each included study, both authors independently extracted and recorded data into data collection forms developed by Cochrane Eyes and Vision. We extracted data on the following topics.

1. Study design and participant characteristics.

2. Method used to generate the random sequence; method of allocation and allocation concealment before randomization; 
blinding of study participants, study personnel, and outcome assessors; number and reason for losses to follow-up in each treatment group; evidence for reporting biases (selective outcome reporting); funding sources; and other potential sources of bias.

3. Data on primary and secondary outcomes for this review.

We used paper data collection forms for duplicate data extraction and resolved discrepancies by consensus. One review author entered data into Cochrane's Review Manager software (RevMan 2014); the other author verified all the data entered. We did not need to contact trial investigators for missing or unclearly reported information for this review; we used the data available in the study reports.

\section{Assessment of risk of bias in included studies}

We examined the following domains to determine the risk of bias for randomized trials included in this review. We assessed each domain as being at 'low risk,' 'high risk,' or 'unclear risk' of bias using criteria listed in Chapter 8 of the Cochrane Handbook of Systematic Reviews of Interventions (Higgins 2011).

- Random sequence generation.

- Whenever authors clearly described the method used to generate the randomization sequence and stated that they used a random number table or a computer-based generation of the random sequence, we considered the study at low risk of bias based on this aspect of selection bias.

- Method of allocation concealment before randomization.

- We considered methods such as central randomization or sequentially numbered, adequately sealed opaque envelopes to confer low risk for this aspect of selection bias.

- Blinding.

- Blinding of participants (infants and children less than four years of age) was not necessary. Blinding of care providers and/or parents would have been difficult or impossible. Thus, we examined study methods to determine if the lack of blinding would have created potential performance bias. Because blinding of personnel who assessed outcomes was possible, we judged a study that blinded outcome assessors as being at low risk of detection bias.

- Incomplete outcome data.

- We examined data reported from included trials on exclusions after randomization, losses to follow-up, and reasons for exclusions and losses to follow-up after randomization in each group. We assessed the amount of missing data and methods used to handle missing data. Whenever the reasons for missing data were likely to be related to the outcome, we classified the study as being at high risk of attrition bias.

- Selective outcome reporting.

- We compared outcomes defined for each study in protocols, clinical trial registrations, design and baseline papers, and similar documents whenever available, assessing consistency of reported outcomes with those specified in such documents. We did not contact authors to obtain trial protocols not otherwise available.

- Other potential sources of bias.

- We documented sources of funding. We considered potential conflicts of interest and obvious study design problems that could have merited a judgement of high risk of bias.

\section{Measures of treatment effect}

For dichotomous outcomes (proportion of participants for whom treatment was successful, who needed secondary procedures, and who experienced complications), we planned to report a risk ratio and $95 \%$ confidence interval. For continuous outcomes, we planned to report mean differences and corresponding 95\% confidence interval (costs of procedures).

\section{Unit of analysis issues}

The included studies enrolled and randomized participants with unilateral or bilateral NLDO. The trial investigators treated both eyes of children with bilateral NLDO with the same intervention, but analyzed the eyes as independent units of analysis. We did not have sufficient data to perform correct within-person analysis (Deeks 2011) and, instead, present the data as presented in the published study reports.

\section{Dealing with missing data}

Included studies reported all desired data. Thus, we did not need to contact the primary investigators of included studies to request details regarding study methods or outcome data. We did not impute data for the purposes of this review.

\section{Assessment of heterogeneity}

We assessed clinical and methodological heterogeneity by examining variability in inclusion/exclusion criteria, characteristics of study participants and interventions, and length of followup in the included studies. We assessed variability in risk of bias parameters to determine presence of methodological heterogeneity. We planned to use the $\mathrm{Chi}^{2}$ test of homogeneity and $1^{2}$ values to identify statistical heterogeneity, but we did not include any meta-analyses in this review.

\section{Assessment of reporting biases}

We assessed selective outcome reporting by comparing outcomes designated with those reported in full-text publications. For trials with multiple publications, including conference abstracts and full-text publications, we examined all available study reports for consistency in description of methods and outcomes. If we had included 10 or more studies in a meta-analysis, we would have examined a funnel plot for evidence of potential publication bias.

\section{Data synthesis}

We determined whether data synthesis was possible by investigating study characteristics for clinical heterogeneity; risk of bias for methodological heterogeneity; and the $\mathrm{Chi}^{2}$ test, I2 statistic, and overlap of confidence intervals for statistical heterogeneity. As long as we did not detect any substantial clinical, methodological, or statistical heterogeneity, we planned to combine data in metaanalysis using a fixed-effect model when there were fewer than three studies providing data and a random-effects model when there were three or more studies. The two studies included in this review had quite different methods, different comparisons and different outcomes, so we were unable to combine data or perform any meta-analyses.

For economic data, we planned to describe costs associated with each treatment group (i.e. costs of surgical procedure and associated medical costs) and provide a narrative summary of costeffectiveness measures including incremental cost-effectiveness 
ratio (ICER). Only one of the included studies reported on the costs of each treatment group, and we provide that information in a narrative format.

\section{Subgroup analysis and investigation of heterogeneity}

If sufficient data had been available, we would have performed subgroup analyses based on whether probing was conducted in the office setting or in an outpatient hospital setting under general anesthesia. One of the studies we included performed probing in the office setting, and the second included study did not report where the probing took place. We also planned to create subgroups based on the age of participating children (less than 18 months of age compared with children 18 months of age or older). In both of the included studies, the children enrolled were less than one year of age, so subgroup analyses for these age subgroups was not possible. For one study, we reported data in subgroups comparing unilateral and bilateral NLDO, as it was presented in the study report.

\section{Sensitivity analysis}

We planned to perform a sensitivity analysis by excluding studies classified as being at high risk of bias for incomplete outcome data and selective outcome reporting; however, with only two included studies and no meta-analyses, this was not necessary. We also planned to examine the effect of excluding industry-funded studies and quasi-randomized trials, but neither of the included studies were industry funded and both employed randomization of participants to study arms.

\section{Summary of findings table}

We created a 'Summary of findings' table that presents the relative and absolute risks of the following outcomes: treatment success, proportion of participants requiring secondary procedures, and complications. We also show mean difference in costeffectiveness. We graded the certainty of the evidence using the GRADE classification (GRADEpro 2014). The factors considered to determine the quality of the evidence were high risk of bias due to limitations in the study design, inconsistency of the results, indirectness of the evidence, imprecision, and publication bias. If an outcome was subject to one or more of these factors, we downgraded the quality of the evidence from high to moderate, low, or very low depending on the number of reasons identified.

\section{RE S U L T S}

\section{Description of studies}

\section{Results of the search}

The electronic searches as of 30 August 2016 yielded 2609 titles and abstracts and 41 records from trial registers (Figure 1 ). Following independent review by two authors, we judged 2637 to be 'not relevant,' leaving 13 total references to be moved into full-text review. Of these 13 references, we deemed 7 (comprised of 6 publications plus 1 trial registry entry) to be eligible for the review. Five of the publications and the trial registry entry all referred to the same trial (PEDIG 2012). The other publication was a single report of another trial (Young 1996). We recorded the study characteristics in the 'Characteristics of included studies' table. We excluded the remaining six studies, documenting our reasons in the 'Characteristics of excluded studies' table. We identified no ongoing trial. 
Figure 1. Study flow diagram.

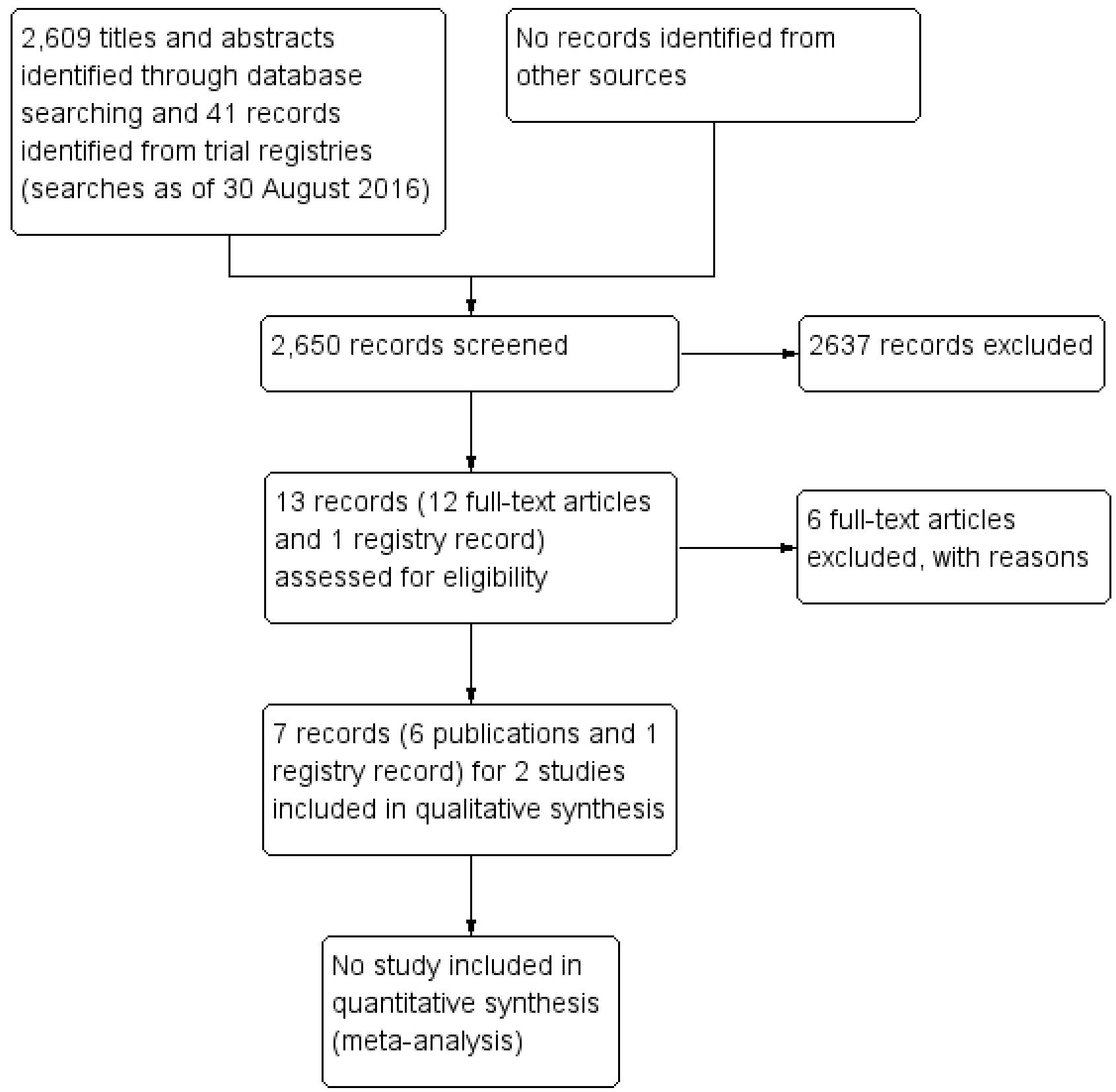

\section{Included studies}

We included two RCTs in this review (the Pediatric Eye Disease Investigator Group, or PEDIG 2012, and Young 1996). Both studies included some participants with unilateral NLDO and some with bilateral NLDO; this review includes 303 eyes of 242 participants.

\section{Types of participants}

Both of the included studies enrolled infants (12 months of age or younger) with congenital NLDO. Participants in the PEDIG 2012 trial were 6 to 10 months old at the time of enrollment and had not had previous surgery for NLDO; a history of NLDO treatment with lacrimal massage, topical antibiotics or steroids, or systemic antibiotics was permitted. Young 1996 described participants' age as "approaching or just after their first birthday," and did not have a history of previous lacrimal procedures. It is unclear whether conservative treatment such as massage and antibiotics were allowed, though it was reported that babies who had persistent conjunctival hyperemia and discharge "except while on antibiotics" were excluded from randomization and probing was advised. PEDIG 2012 reported that symptoms of NLDO should have started before the age of six months. For both studies participants with bilateral NLDO had both eyes assigned to the same treatment; however, eyes were analyzed as independent units of analysis.

\section{Types of interventions}

Both of the studies had one treatment arm that was considered 'immediate' probing and an observation arm that prescribed probing only when spontaneous resolution had not occurred by a specified follow-up time point (delayed probing arm). In 
PEDIG 2012, participants were randomized to immediate office probing using topical anesthesia and a gentle restraint or to six months of observation and when necessary, deferred facility probing for unresolved cases. During the six-month observation period, participants' parents received instructions to provide lacrimal massage whenever discharge was present and to use antibiotic eyedrops when discharge was purulent. After the sixmonth observation period, 37 eyes ( $28 \%$ of eyes randomized to the observation/deferred arm) underwent deferred-facility probing. The intervention plan was slightly different in Young 1996; instead of 6 months of observation as in PEDIG 2012, in Young 1996 probing was delayed until the child reached 24 months of age. Young 1996 performed immediate probing under general anesthesia; authors did not report whether the observation/delayed probing group received massage and antibiotics in cases of discharge.

\section{Type of outcomes}

For both included studies, the resolution of NLDO signs and symptoms was a primary outcome. PEDIG 2012 described treatment success as the absence of clinical signs and symptoms of NLDO such as epiphora, increased tear lake, and mucus discharge. It is important to note that PEDIG 2012 was planned to be a costeffectiveness study; treatment success was associated with the end of the cost of treatment. Young 1996 used the term 'cure,' which was defined as a complete or near complete remission of symptoms and signs including a normal fluorescein dye disappearance test. Though these resolution definitions are similar, the time points when the two studies measured them were quite different. PEDIG 2012 reported on some of the participants for treatment success six months after enrollment, when they would have been 12 to 16 months of age (depending on age at enrollment), and all participants at age 18 months, which was 9 to 12 months after randomization. PEDIG 2012 additionally reported data for participants with unilateral and bilateral NLDO in separate study reports; therefore, chose to report this data separately as well as provide a combined estimate. In Young 1996, participants enrolled at around one year of age (and were probed at 12 to 14 months), and investigators assessed 'cure' at age 15 months, which was only about 1 to 3 months after enrollment, compared with 6 or 9 to 12 months after enrollment in the PEDIG 2012 study.

\section{Excluded studies}

We excluded six studies and documented reasons for exclusion in the 'Characteristics of excluded studies' table. Five studies were not RCTs (one case series: Chaabouni 1993; one that provided various treatments to patients for NLDO in a particular order in which they only received probing if all previous procedures had failed: Ekinciler 1994; and three that described probing surgery in a narrative format: Hernandez 1967; Ishikawa 1990; Robb 1985). We excluded one study that compared probing to a another surgical procedure (bicanalicular silastic intubation) for treating NLDO (AlFaky 2015).

\section{Risk of bias in included studies}

We assessed the risk of bias for the two individual studies included in the review and summarize our judgements in Figure 2. We provide justification of our classifications of risk of bias for each study in the 'Characteristics of included studies' tables. 
Figure 2. Risk of bias summary: review authors' judgements about each risk of bias item for each included study.

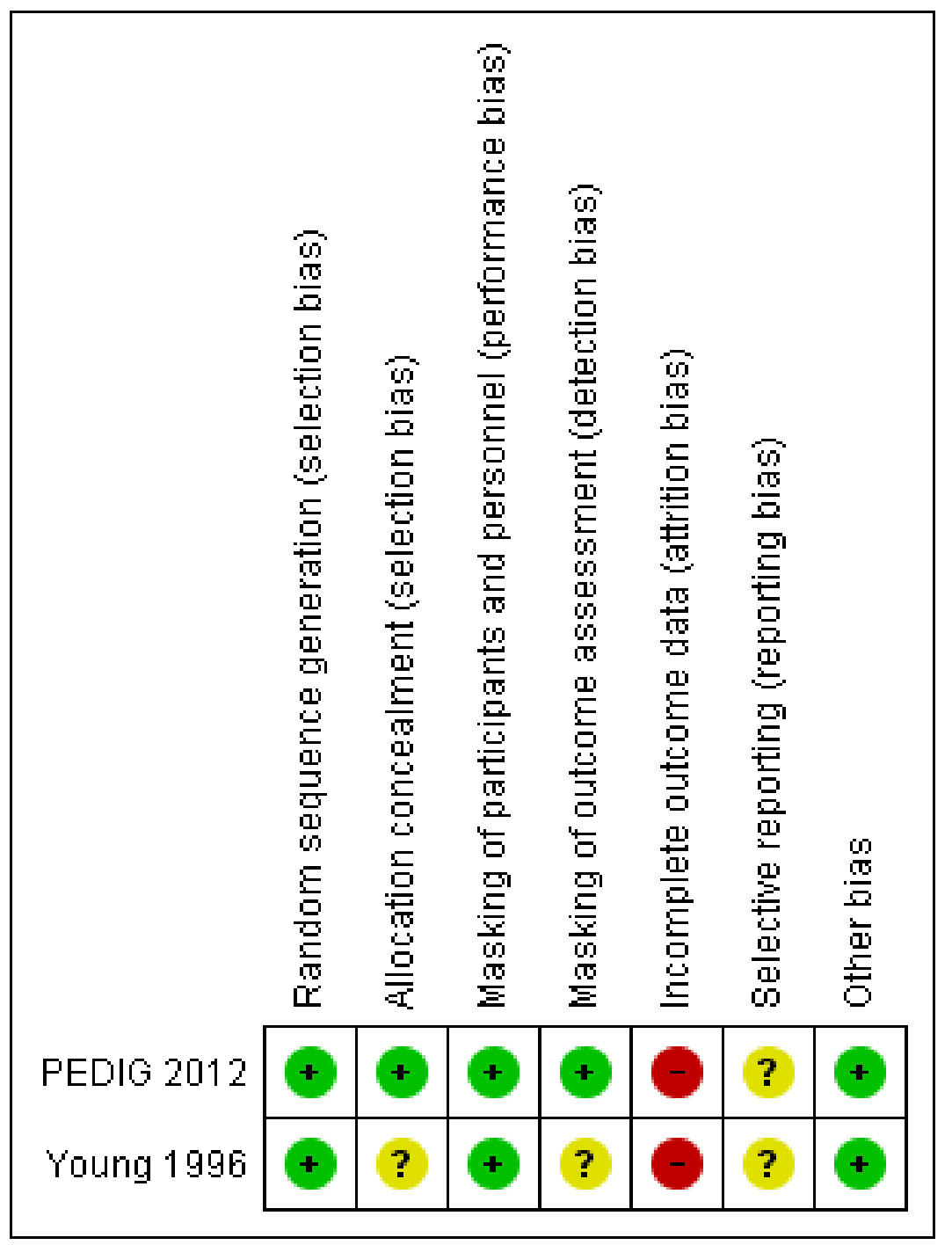

\section{Allocation}

Authors described both studies as parallel-group randomized controlled trials. The studies reported adequate methods for random sequence generation, both used random number tables; thus, we judged both studies to be at low risk of bias with respect to random sequence generation. PEDIG 2012 concealed allocations before randomization as study staff entered a participant's data onto a website that used a permutated block design to make the random assignment; thus we assessed the study to be at low risk of bias with respect to allocation concealment. Young 1996 did not describe allocation concealment, meriting a judgement of unclear risk of bias for this aspect of selection bias.

\section{Masking (performance bias and detection bias)}

Due to the nature of the treatments and comparisons, participants (their parents) and clinical personnel could not be masked. The primary outcome of this review was treatment success, defined as absence of clinical signs and symptoms of NLDO. Study visits were scheduled the same regardless of treatment assignment, and follow-up was the same. We do not anticipate that the treatment effect would be influenced by the knowledge of the treatment group; we therefore judged the studies to be at low risk of performance bias.

PEDIG 2012 reported that the outcome assessor was masked, resulting in our classification of it as being at low risk of detection bias. Young 1996 did not provide enough information regarding masking of the outcome assessor to classify the trial as being at low risk or high risk of detection bias; thus, we assigned an unclear risk of detection bias for this domain.

\section{Incomplete outcome data}

We judged Young 1996 to be at unclear risk of attrition bias and PEDIG 2012 to be at high risk of attrition bias. Young 1996 reported incomplete outcome data for six participants, who they excluded from the analysis. We could not ascertain whether these six participants were from the RCT or the companion observational study whose findings were also included in the study report. In PEDIG 2012, the losses to follow-up were $12 \%$ in the immediate probing group and $10 \%$ in the observation/deferred probing group, without reasons provided. 


\section{Selective reporting}

The PEDIG 2012 protocol was available online, and authors reported the outcomes they had pre-specified in the protocol. However, data from this study was reported in two separate study reports, one including only participants with unilateral NLDO and one including participants with bilateral NLDO. This separated analysis was not described in the protocol and was therefore an ad hoc decision. Since the study had not been powered to treat the two subgroups as separate analyses, we judged the risk of reporting bias to be unclear. We did not find a protocol for Young 1996; therefore we judged this study to be at unclear risk of reporting bias.

\section{Other potential sources of bias}

The PEDIG 2012 study indicated that industry provided some eyedrops used in the study, but since the eyedrops were not one of the interventions being compared, we did not consider this minor involvement of industry to be a source of bias. We rated PEDIG 2012 as being at low risk of other bias. We did not identify any other potential sources of bias in Young 1996.

\section{Effects of interventions}

\section{See: Summary of findings for the main comparison}

\section{Primary outcome: treatment success at six months}

In our protocol we defined treatment success, our primary outcome, as "the absence of clinical signs and symptoms, including epiphora and mucous discharge" (Petris 2014). One of the studies in this review used a similar definition; PEDIG 2012 described treatment success as "the absence of clinical signs and symptoms of NLDO such as epiphora, increased tear lake, and mucus discharge." The other study did not use the term 'treatment success,' but rather used the term 'cure,' which was similar to the definitions of treatment success but included the additional requirement that participants have "a normal fluorescein dye disappearance test" as well as "a complete or near complete remission of symptoms and signs" (Young 1996).

PEDIG 2012 analyzed treatment success at six months after randomization for the participants who were randomized to observation/deferred probing. At this time point, NLDO resolved without surgery in 77 out of 117 eyes (66\%), which included resolution in 44 participants with unilateral NLDO $(66 \%$ of those who completed the 6-month visit), resolution in both eyes for 14 participants with bilateral NLDO (56\% of those who completed the 6-month visit), and resolution in only one eye for 5 participants with bilateral NLDO ( $20 \%$ of those who completed the 6 -month visit). Forty out of 117 eyes (34\%) did not resolve without surgery in the observation/deferred probing after six months. Data extracted from a table in Lee 2012 (one of the PEDIG 2012 reports that included only participants with unilateral NLDO), showed a six-month success rate in $77 \%$ (63/82 participants) of the immediate office probing group and $54 \%(44 / 81)$ in the deferred facility probing group. For these participants, those randomized to immediate probing had better treatment success at 6 months (RR $1.41,95 \% \mathrm{Cl} 1.12$ to 1.78 ). We judged the certainty of evidence for this outcome as moderate; we downgraded one level due to risk of attrition bias. Outcomes were otherwise reported at 9 to 12 months after probing. Treatment success at 6 months was not an outcome in the Young 1996 study.

\section{Secondary outcomes}

\section{Treatment success at other time points}

PEDIG 2012 reported on treatment success for participants at 18 months of age, which was 9 to 12 months after randomization, depending on age at enrollment. Authors reported results separately for participants with unilateral and bilateral NLDO. This was an ad hoc decision that was not reported in the trial protocol. Among the subgroup participants with unilateral NLDO, there was a higher percent of participants who had treatment success in the immediate probing group compared with the observation/ deferred probing group (92\% versus $82 \%$, respectively; RR 1.13 , $95 \% \mathrm{Cl} 0.99$ to 1.28; Analysis 1.1.1), and among the subgroup of participants with bilateral NLDO there was a higher percent of participants who has treatment success in the observation/ deferred group compared with the immediate probing group $71 \%$ versus $82 \%$, respectively; RR $0.86,95 \% \mathrm{Cl} 0.70$ to 1.06 ; Analysis 1.1.2), though there was uncertainty in both estimates. Among participants in the immediate probing group with bilateral NLDO, $19 / 29(66 \%)$ experienced treatment success in both eyes and 3/29 $(10 \%)$ in one eye. In the observation/deferred treatment group, $19 / 25(76 \%)$ had resolution in both eyes and $3 / 25(12 \%)$ in one eye. There was moderately high attrition in this study (at least $10 \%$ of participants in both study arms did not attend the 18 month outcome evaluation). We judged these estimated outcome rates to have very low-certainty; we downgraded the quality of the evidence one level each due to risk of attrition bias from attrition bias, inconsistency in the direction of the effect estimates, and imprecision due to an ad hoc subgroup analysis for which the study was not adequately powered.

Young 1996 reported on 'cure' rate when participants were aged 15 months old. For those probed at age 12 to 14 months (immediate probing), $8 / 10$ (80\%) were cured compared with $5 / 16(31 \%)$ in the delayed probing group (RR 2.56, 95\% $\mathrm{Cl} 1.16$ to 5.64 ). In the delayed probing group, participants were to receive probing at 24 months of age if resolution had not occurred. By age 24 months, two more participants had spontaneous resolution of the NLDO $(7 / 16,43 \%)$. We judged these estimates to be of low-certainty. We downgraded two levels due imprecision from to the small number of participants $(\mathrm{N}=26)$ in the randomized trial portion of this study and for high risk of attrition bias, as outcomes were assessed at different followup time points for participants in the immediate probing group compared with the observation/deferred probing group.

\section{Proportion of participants requiring secondary procedures}

In the PEDIG 2012 study, some participants who were randomized to immediate office probing required reoperation at a later time point. Reoperation took place in a surgical facility under general anesthesia in $7 / 82$ participants (9\%) with persistent unilateral NLDO and in 4/31 (13\%) with bilateral NLDO. The second surgical procedures took place before participants reached 18 months of age.

\section{Cost-effectiveness}

PEDIG 2012 was designed as a cost-effectiveness study. For participants with unilateral NLDO the mean cost of treatment was USD 562 for those randomized to immediate probing, compared with USD 701 for those randomized to observation/deferred probing (difference USD 139, 95\% CI USD -377 to 94). When antibiotics were prescribed, the average cost of treatment was 
USD 551 in the immediate office-probing group and USD 652 in the observation/deferred facility probing group, provided that the less costly antibiotic tobramycin was given instead of the more costly moxifloxacin. We considered the quality of the evidence to be moderate; we downgraded one level for publication bias, because the other study in this review (Young 1996) did not report on this outcome, and the estimate may change with further research.

\section{Complications}

We examined complications and adverse events associated with treatment for NLDO, including bleeding, injury to the nasolacrimal system or the eye, canalicular stenosis (narrowing of the nasolacrimal duct due to fibrosis), and other complications of probing. PEDIG 2012 reported that there were no complications following surgery, including in the surgeries for participants from the observation/deferred probing group who were treated because they did not have resolution and in reoperations in either group. Another report of data from this study shows there are no adverse events (including no serious adverse events). Young 1996 reported that bleeding from the punctum occurred in $20 \%$ of all probings, but this percentage was based on participants in both the RCT and those who were part of the non-randomized study also in this report. Young 1996 also reported that no serious infective complication occurred in the study.

\section{DISCUSSION}

\section{Summary of main results}

We included two RCTs that reported on 303 eyes of 242 participants in this review. The studies enrolled children with unilateral or bilateral congenital nasolacrimal duct obstruction. For both included studies, the intervention was an immediate office-based probing to remove the duct obstruction versus observation until the child reached a specified age to allow for resolution without surgery and probing for those without resolution. One included study was undertaken as a cost-effectiveness comparison, but both used 'treatment success' or 'cure' as the primary clinical outcome of interest.

Neither of the studies compared treatment success at six months, our primary outcome, for all participants. One study reported on resolution of NLDO by six months after randomization for only participants in the observation/deferred probing group. We obtained six-month resolution data from a figure for some of the participants in the immediate probing arm, but this did not include the entire cohort. Thus, we were only able to compare six-month resolution in the two study arms for participants with unilateral NLDO. A higher proportion of participants in the immediate probing arm had treatment success compared with the observation/ deferred probing arm (RR $1.41,95 \% \mathrm{Cl} 1.12$ to 1.78). The same study reported outcomes within subgroups of children with unilateral and bilateral NLDO at 18 months of age; the proportion with treatment success for children at 18 months was higher in the group treated with immediate office-based probing compared with observation/deferred probing for participants with unilateral NLDO, but in participants with bilateral NLDO, treatment success was more common in the group randomized to observation/ deferred probing. The authors suggested that potential reasons for a lower success rate with immediate probing for children with bilateral NLDO compared with unilateral NLDO is that with a conscious child, the increased time required to probe both eyes could lead to a more hurried procedure or resistance from the child undergoing probing in the second eye (PEDIG 2012). In the statistical analysis there was uncertainty as to whether treatment success was more likely in the immediate probing group versus the deferred probing group. This uncertainty was present for combined data and when analyzed as subgroups of participants with unilateral and bilateral NLDO. We considered there to be moderate-certainty evidence from this study, as there were problems with attrition ( $10 \%$ of each group was lost to followup).

From one small study there was weak (low-certainty) evidence that children in the immediate probing group were more likely to be cured within a month after surgery compared to children who were randomized to deferred probing at 15 months (RR 2.56, 95\% Cl 1.16 to 5.64). The evidence for the estimates again was considered weak (low-certainty) due to imprecision arising from the small sample size and risk of attrition bias due to participants' outcomes being assessed at different time points depending on the group to which they had been randomized.

Just one of the included studies reported on the cost-effectiveness of immediate probing versus deferred probing. The mean cost of treatment for immediate probing was less than for deferred probing; however, there is uncertainty as to whether there is a true cost difference (mean difference USD - 139, 95\% CI USD - 377 to 94). A significant portion of the cost of treating NLDO comes from the use of antibiotics; the authors suggested that a less expensive antibiotic could have reduced costs in both immediate and deferred probing groups.

\section{Overall completeness and applicability of evidence}

We identified only two studies that examined probing for nasolacrimal duct obstruction. The trial with a larger sample size that analyzed 254 eyes, PEDIG 2012, had unexplained losses to follow-up of over $10 \%$ in each intervention group. We were unable to perform meta-analyses with the data from these studies because the time points of outcomes were too different. Additonally, one study made use of non-surgical interventions during the observation/deferred waiting period, but it is unclear whether the other did as well. The massage and antibiotics permitted by the PEDIG 2012 protocol were to be "as-needed," meaning two different children randomized to that arm could have different treatment experiences during the observation period. PEDIG 2012 additionally allowed for enrollment of children who had a history of NLDO treatment with lacrimal massage, topical antibiotics or steroids, or systemic antibiotics; thus, children could have been enrolled with different treatment histories. PEDIG 2012 reported data for participants with unilateral and bilateral NLDO in separate study reports and we chose to report this data separately as a combined estimate, though we had not originally planned to do this type of subgroup analysis. The differences between resolution of NLDO in participants with unilateral and bilateral disease is noteworthy, and would not have been identified if the study authors had only reported data for all participants. It is unclear whether participants in Young 1996 were allowed to have had conservative treatment prior to enrollment. This review also did not include any studies that compared immediate probing with massage alone or antibiotics alone, only the "as-needed" combination, as mentioned above. None of the study reports for the included studies stratified resolution outcomes by surgeon, so it is possible there could be an undetected surgeon effect, in one 
or both of the included studies; one surgeon may have had more successful probing outcomes than another surgeon based on skill or technique.

\section{Quality of the evidence}

Besides the moderate attrition in the PEDIG 2012, we judged it to have an overall low risk of bias in other domains. The investigators published the protocol online and though they did report on all pre-specified outcomes, they made a decision to publish data in two separate study reports, dividing the participants with unilateral NLDO and bilateral NLDO. We believed this type of ad hoc subgroup analysis would result in an effect estimate for which the study was inadequately powered and affect the precision of the estimate, so we judged this to be of unclear risk of bias. Young 1996 reported details of study methods less clearly. We judged the study to have high risk of attrition bias because children in each intervention group had outcome assessments performed at different follow-up times.

For the primary outcome of resolution of NLDO, we did not judge any of the outcomes from these studies as providing high-certainty; our confidence in the outcome effect estimates is limited. More data from large studies would likely change these estimates of outcome rates.

\section{Potential biases in the review process}

In order to minimize bias in the review process, we followed the standard methods recommended by Cochrane in completing this review. However, it is possible that we failed to identify other RCTs that would have been eligible for this review.

\section{Agreements and disagreements with other studies or reviews}

A older cohort study of 4792 infants with epiphora and 964 with evidence of defective lacrimal drainage reported that by one year old, $96 \%$ no longer had symptoms (MacEwen 1991). Neither of the studies included in this review had quite as high a rate of resolution (PEDIG 2012 with approximately $82 \%$ resolution in the deferred probing by age 18 months and Young 1996 with only 43\% in the deferred probing group with resolution by 24 months). It is possible that participants enrolled in the RCTs that we included in this review had more serious symptoms or more severe blockage, prompting parents to seek out medical care for their child. In a nonrandomized observational study of probing for nasolacrimal duct obstruction conducted by the Pediatric Eye Disease Investigator Group conducted in 2005 and 2006, authors reported that the proportion of eyes treated successfully with probing was 78\% (95\% $\mathrm{Cl} 75 \%$ to $81 \%$ ), which is similar to the outcomes we saw in the immediate probing groups in the RCTs included in this review (PEDIG 2008). Some studies have shown that late probing has a higher failure rate than early probing (Nelson 1985; Paul 1985; Paul 1994; Petersen 1978; Stager 1992). In the observational study, this pattern held: there was a $79 \%$ success rate for those aged 12 months to $<24$ months ( 421 eyes), $79 \%$ for 24 to $<36$ months ( 37 eyes), and $56 \%$ for 36 months to $<48$ months ( 11 eyes) (PEDIG 2008). Similarly to PEDIG 2012, treatment success was lower in eyes of children with bilateral disease in this multicenter observational study.

A recently published systematic review on treatments for congenital NLDO included the PEDIG 2012 study as two studies (counting the unilateral and bilateral report each as its own study). They also reported that immediate probing had similar rates of success as observation/deferred probing (Lin 2016).

\section{AUTHORS' CONCLUSIONS}

\section{Implications for practice}

At present, the decision to probe is usually based on severity of symptoms and the surgeons' recommendation to parents. This review aimed to assess the evidence for probing for congenital nasolacrimal duct obstruction. We found that the effects and costs of immediate versus deferred probing for NLDO are uncertain for most outcomes. We found immediate probing may be a successful intervention in children with unilateral NLDO versus deferred probing at six months. This review could not determine the optimal timing of probing early ( $<12$ months) versus late ( $>12$ months). In summary, few complications were reported for immediate versus deferred probing and immediate probing may be lower in cost, though there was uncertainty in the cost difference. Children who have unilateral NLDO may benefit from immediate office probing, although evidence for this suggestion is weak.

\section{Implications for research}

Larger randomized trials comparing the two approaches investigated in this review are needed to provide robust estimates of outcomes. Future studies of probing for NLDO should adhere to strict protocols for adjunctive treatment permitted during the period of observation before deferred probing to assure similar management of children assigned to immediate and deferred groups.

\section{ACKN OWLEDGEMENTS}

We acknowledge:

- Lori Rosman for devising and running the electronic search strategies;

- Anupa Shah, Managing Editor for CEV (Cochrane Eyes and Vision); and

- Xuan Hui and Elizabeth Clearfield at the CEV US Project for support and guidance in completing this review. 


\section{RE F E R E N C E S}

\section{References to studies included in this review}

\section{PEDIG 2012 \{published data only\}}

Lee KA, Chandler DL, Repka MX, Beck RW, Foster NC, Frick KD, et al. A randomized trial comparing cost-effectiveness of immediate office probing versus observation with deferred facility probing for unilateral congenital nasolacrimal duct obstruction. Journal of AAPOS 2012;16(1):e6.

Lee KA, Chandler DL, Repka MX, Melia M, Beck RW, Summers CG, et al. A comparison of treatment approaches for bilateral congenital nasolacrimal duct obstruction. American Journal of Ophthalmology 2013;156(5):1045-50.

* Pediatric Eye Disease Investigator Group. A randomized trial comparing the cost-effectiveness of 2 approaches for treating unilateral nasolacrimal duct obstruction. Archives of Ophthalmology 2012;130(12):1525-33.

Pediatric Eye Disease Investigator Group. Resolution of congenital nasolacrimal duct obstruction with nonsurgical management. JAMA Ophthalmology 2012;130(6):730-4.

Petersen DB, Chandler DL, Repka MX, Beck RW, Crouch ER, Lee $K A$, et al. Resolution of nasolacrimal duct obstruction with nonsurgical management in children 6 to $<10$ months old. Journal of AAPOS 2012;16(1):e6.

\section{Young 1996 \{published data only\}}

Young JD, MacEwen CJ, Ogston SA. Congenital nasolacrimal duct obstruction in the second year of life: a multicentre trial of management. Eye 1996;10(4):485-91.

\section{References to studies excluded from this review}

Al-Faky 2015 \{published data only\}

Al-Faky YH, Mousa A, Kalantan $\mathrm{H}$, Al-Otaibi A, Alodan H, Alsuhaibani $\mathrm{AH}$. A prospective, randomised comparison of probing versus bicanalicular silastic intubation for congenital nasolacrimal duct obstruction. British Journal of Ophthalmology 2015;99(2):246-50.

\section{Chaabouni 1993 \{published data only\}}

Chaabouni M, Zayani A, Chebihi S, Guiaa R, Ben HH. Congenital obstruction of lacrimal ducts in 578 children. Archives Françaises de Pédiatrie 1993;50(2):107-9.

\section{Ekinciler 1994 \{published data only\}}

Ekinciler OF, Doğan H, Tatlişen N, Karaküçük S. Congenital nasolacrimal duct obstruction in Kayseri, Turkey. Turkish Journal of Pediatrics 1994;36(1):21-33.

\section{Hernandez 1967 \{published data only\}}

Hernandez JA. Treatment of congenital lacrimal duct obstruction [Actualizacion del tratamiento de la obstruccion congenita de las via lagrimales]. Archivos de Oftalmologia de Buenos Aires 1967;42(4):59-61.
Ishikawa 1990 \{published data only\}

Ishikawa C, Tanaka H, Toibana M. Early probing for congenital nasolacrimal duct obstruction. Folia Ophthalmologica Japonica 1990;41(5):940-4

Robb 1985 \{published data only\}

Robb RM. Treatment of congenital nasolacrimal system obstruction. Journal of Pediatric Ophthalmology and Strabismus 1985;22(1):36-7.

\section{Additional references}

\section{Cassady 1948}

Cassady JV. Dacryocystitis of infancy. American Journal of Ophthalmology 1948;31(7):773-80.

\section{Cassady 1952}

Cassady JV. Developmental anatomy of nasolacrimal duct. A.M.A. Archives of Ophthalmology 1952;47(2):141-58.

\section{Deeks 2011}

Deeks JJ, Higgins JP, Altman DG editor(s). Chapter 9: Analysing data and undertaking meta-analyses. In: Higgins JP, Green $S$ editor(s). Cochrane Handbook for Systematic Reviews of Interventions Version 5.1.0 (updated March 2011). The Cochrane Collaboration, 2011. Available from handbook.cochrane.org.

\section{Ffookes 1962}

Ffookes 00. Dacryocystitis in infancy. British Journal of Ophthalmology 1962;46(7):422-34.

\section{GRADEpro 2014 [Computer program]}

GRADE Working Group, McMaster University. GRADEpro GDT. Version accessed 11 January 2017. Hamilton (ON): GRADE Working Group, McMaster University, 2014.

\section{Guerry 1948}

Guerry D, Kendig EL. Congenital impatency of the nasolacrimal duct. Archives of Ophthalmology 1948;39(2):193-204.

\section{Higgins 2011}

Higgins JP, Altman DG, Sterne JAC editor(s). Chapter 8: Assessing risk of bias in included studies. In: Higgins JP, Green S editor(s). Cochrane Handbook for Systematic Reviews of Interventions Version 5.1.0 (updated March 2011). The Cochrane Collaboration, 2011. Available from handbook.cochrane.org.

\section{Lin 2016}

Lin AE, Chang YC, Lin MY, Tam KW, Shen YD. Comparison of treatment for congenital nasolacrimal duct obstruction: a systematic review and meta-analysis. Canadian Journal of Ophthalmology 2016;51(1):34-40.

\section{MacEwen 1991}

MacEwen CJ, Young JD. Epiphora during the first year of life. Eye 1991;5(Pt 5):596-600. 


\section{Nelson 1985}

Nelson LB, Calhoun JH, Menduke H. Medical management of congenital nasolacrimal duct obstruction. Ophthalmology 1985;92(9):1187-90.

\section{Paul 1985}

Paul TO. Medical management of congenital nasolacrimal duct obstruction. Journal of Pediatric Ophthalmology and Strabismus 1985;22(2):68-70.

\section{Paul 1994}

Paul TO, Shepherd R. Congenital nasolacrimal duct obstruction: natural history and the timing of optimal intervention. Journal of Pediatric Ophthalmology and Strabismus 1994;31(6):362-7.

\section{PEDIG 2008}

Pediatric Eye Disease Investigator Group. Primary treatment of nasolacrimal duct obstruction with probing in children less than four years old. Ophthalmology 2008;115(3):577-584.e3.

\section{Petersen 1978}

Petersen RA, Robb RM. The natural course of congenital obstruction of the nasolacrimal duct. Journal of Pediatric Ophthalmology and Strabismus 1978;15(4):246-50.

\section{RevMan 2014 [Computer program]}

Nordic Cochrane Centre, The Cochrane Collaboration. Review Manager (RevMan). Version 5.3. Copenhagen: Nordic Cochrane Centre, The Cochrane Collaboration, 2014.

\section{Schnall 2013}

Schnall BM. Pediatric nasolacrimal duct obstruction. Current Opinion in Ophthalmology 2013;24(5):421-4.

\section{Sevel 1981}

Sevel D. Development and congenital abnormalities of the nasolacrimal apparatus. Journal of Pediatric Ophthalmology and Strabismus 1981;18(5):13-9.

\section{Shekunov 2011}

Shekunov J, Griepentrog GJ, Diehl N, Mohney BJ. Prevalence and clinical characteristics of congenital dacryocystocele. Journal of AAPOS 2011;14(5):417-20.

\section{Stager 1992}

Stager D, Baker JD, Frey T, Weakley DR, Birch, EE. Office probing of congenital nasolacrimal duct obstruction. Ophthalmic Surgery 1992;23(7):482-4.

\section{Tan 2001}

Tan AD, Rubin PA, Sutula FC, Remulla HD. Congenital nasolacrimal duct obstruction. International Ophthalmology Clinics 2001;41(4):57-69.

\section{Wesley 1985}

Wesley RE. Inferior turbinate fracture in the treatment of congenital nasolacrimal duct obstruction and congenital nasolacrimal duct anomaly. Ophthalmic Surgery 1985;16(6):368-71.

\section{References to other published versions of this review Petris 2014}

Petris C, Liu D. Probing for congenital nasolacrimal duct obstruction. Cochrane Database of Systematic Reviews 2014, Issue 5. [DOI: 10.1002/14651858.CD011109]

* Indicates the major publication for the study

\section{CHARACTERISTICS OF STUDIES}

Characteristics of included studies [ordered by study ID]

PEDIG 2012

Methods

Study design: parallel-group randomized controlled trial

Reported power calculation: yes, $80 \%$

Unusual study design? Some of the participants had unilateral NLDO while others had bilateral NLDO. Participants with bilateral NLDO were randomized to the same intervention for both eyes.

Participants Country: USA

Age: unilateral immediate probing mean age $=7.6 \pm 1.2$ months, unilateral observation/deferred probing mean age $=7.8 \pm 1.3$ months, bilateral immediate probing mean age $=7.8 \pm 1.4$ months, bilateral observation/deferred probing mean age $=7.5 \pm 1.2$ months

Sex: unilateral probing $32(39 \%)$ girls and $50(61 \%)$ boys, unilateral observation/deferred probing 42 (52\%) girls and 39 (48\%) boys, bilateral probing 13 (42\%) girls and 18 (58\%) boys, bilateral observation/deferred probing $11(42 \%)$ girls and $15(58 \%)$ boys

Inclusion criteria: "Major eligibility criteria included age 6 to less than 10 months, onset of NLDO symptoms before age 6 months, presence of at least 1 clinical sign of NLDO (epiphora, increased tear 
lake, and mucous discharge) in the absence of an upper respiratory infection or ocular surface irritation, no prior NLD surgery, and at least 1 patent punctum in the study eye."

Exclusion criteria: "The study excluded children with Down syndrome or craniofacial anomalies."

Equivalence of baseline characteristics: not reported

Number randomized:

Total: 220 (163 participants with unilateral NLDO and 57 with bilateral NLDO)

Per group: immediate office-based probing: 113 (82 unilateral, 31 bilateral); observation/deferred facility-based probing: 107 (81 unilateral, 26 bilateral)

\section{Exclusions after randomization: none}

Losses to follow-up: 9 immediate probing dropped; 14 observation/deferred dropped at 6 months visit Number analyzed:

Total: 140 unilateral

Per group: immediate office-based probing: 73; observation/deferred facility-based probing: 67

Unit of analysis: some of the participants had unilateral NLDO while others had bilateral NLDO. Sometimes results were presented on the participant level and sometimes on the eye level.

\section{Interventions Intervention 1: immediate office-based probing}

Intervention 2: 6 months of observation followed by facility-based probing if needed (lacrimal massage as indicated: performed twice daily when discharge was present, and antibiotic eyedrops as indicated: tobramycin sulfate $0.3 \%$ or moxifloxacin hydrochloride $0.5 \%$ when discharge was purulent)

Length of follow-up: until age 18 months

Outcomes

Primary outcome, as defined in study reports: treatment success, defined as the absence of clinical signs of NLDO assessed by a blinded examiner at 6 months

Secondary outcomes, as defined in study reports: secondary procedure performed within six months

Adverse events reported: yes, "No complications were reported for any surgery."

Intervals at which outcomes assessed: 6 months after randomization, at age 18 months

Funding sources: "This study was supported by grants EY011751 and EY018810 from the National Eye Institute of the National Institutes of Health, Department of Health and Human Services. Alcon Laboratories, Inc provided antibiotic eyedrops and antibiotic/corticosteroid eyedrops at no cost to the study. The sole purpose was to standardize the drugs used in the study; no comparisons were made between the drugs."

Disclosures of interest: "Dr. Lee received support for travel to meetings for the study of other purposes and payment for writing or reviewing the manuscript from the Jaeb Center for Health Research. Ms. Chandler's institution received money from a grant from the National Eye Institute. Dr. Repka's institution received a grant from the National Eye Institute and consultancy for American Academy of Ophthalmology. His institution also received money for support for travel to meetings for the study of other purposes, fees for participation in review activities, such as data monitoring boards, statistical analysis, etc, and payment for writing or reviewing the manuscript from the Jaeb Center for Health Research. Ms. Melia's institution received a grant from the National Eye Institute and was paid for a board membership (Thomas Jefferson University; Alimera Science) and employment by the Jaeb Center for Health Research. Dr. Frick received consulting fees or honoraria from the Jaeb Center for Health Research. Dr. Beck's institution received a grant from the National Eye Institute. Dr. Summers received consultancy fees from BioMarin and Plancon. Ms. Foster's institution received a grant from the National Eye Institute. Mr. Kraker's institution received money from a National Eye Institute grant." 
Reported subgroup analyses: yes (separate reports for patients with unilateral vs bilateral NLDO)

Trial registration: NCT00780741

\section{Risk of bias}

\begin{tabular}{|c|c|c|}
\hline Bias & Authors' judgement & Support for judgement \\
\hline $\begin{array}{l}\text { Random sequence genera- } \\
\text { tion (selection bias) }\end{array}$ & Low risk & $\begin{array}{l}\text { "Clinic personnel entered data on the PEDIG website to randomly assign each } \\
\text { patient (using a permuted block design stratified by site) with equal probabili- } \\
\text { ty to } 1 \text { of } 2 \text { treatment groups" }\end{array}$ \\
\hline $\begin{array}{l}\text { Allocation concealment } \\
\text { (selection bias) }\end{array}$ & Low risk & $\begin{array}{l}\text { "Clinic personnel entered data on the PEDIG website to randomly assign each } \\
\text { patient (using a permuted block design stratified by site) with equal probabili- } \\
\text { ty to } 1 \text { of } 2 \text { treatment groups" }\end{array}$ \\
\hline $\begin{array}{l}\text { Masking of participants } \\
\text { and personnel (perfor- } \\
\text { mance bias) }\end{array}$ & Low risk & $\begin{array}{l}\text { The participants and personnel cannot be blinded because of the nature of the } \\
\text { treatments, but we do not suspect performance bias for the primary outcome. }\end{array}$ \\
\hline $\begin{array}{l}\text { Masking of outcome as- } \\
\text { sessment (detection bias) }\end{array}$ & Low risk & $\begin{array}{l}\text { "The primary efficacy outcome was treatment success, defined as the absence } \\
\text { of clinical signs of NLDO assessed by a masked examiner at the age } 18 \text { months } \\
\text { visit." }\end{array}$ \\
\hline $\begin{array}{l}\text { Incomplete outcome data } \\
\text { (attrition bias) } \\
\text { All outcomes }\end{array}$ & High risk & $\begin{array}{l}\text { Reasons for loss of follow-up were not clearly stated. Over } 10 \% \text { losses of fol- } \\
\text { low-up in each treatment group - } 100 / 113(88 \%) \text { in the immediate probing } \\
\text { group and } 96 / 107(90 \%) \text { in the deferred probing group completed the 18- } \\
\text { month primary outcome examination }\end{array}$ \\
\hline $\begin{array}{l}\text { Selective reporting (re- } \\
\text { porting bias) }\end{array}$ & Unclear risk & $\begin{array}{l}\text { The protocol is available and pre-specified outcomes have been reported. } \\
\text { However, data analysis was reported separately for participants with unilater- } \\
\text { al and bilateral NLDO, but this analysis plan was not described in the protocol. }\end{array}$ \\
\hline Other bias & Low risk & None \\
\hline
\end{tabular}

Young 1996

Methods Study design: randomized controlled trial with an additional observational study group

\section{Reported power calculation: no}

Unusual study design (any issues with study design)? Infants who did not have informed consent given were managed according to each clinician's current practice, and these data were analyzed as an observational study; a total of 101 infants were enrolled, 22 were randomized, 73 were non-randomized (observational group), 6 were excluded due to incomplete data or loss to follow-up, but it was not explicitly stated which group these 6 infants belonged to.

Country: United Kingdom (seven centers)
Age: mean age not provided, but infants in the study were all "approaching or just after their first birth-
day"
Sex: not reported


Inclusion criteria: presenting within the time limits with no medical contraindication, congenital NLDO with a history of epiphora and/or discharge starting within 3 months of birth and an abnormal fluorescein dye disappearance test (FDDT)

Exclusion criteria: history of previous lacrimal procedures

Equivalence of baseline characteristics: not reported

\section{Number randomized:}

Total: 26 eyes ( 22 infants)

Per group: 10 eyes probed at age 12-14 months; 16 eyes no treatment to 24 months

Exclusions after randomization: "6 were excluded because of incomplete data or lost follow-up"; it is not clearly stated if the 6 infants were in the randomization group or the observation group

Losses to follow-up: "6 were excluded because of incomplete data or lost follow-up"; it is not clearly stated if the 6 infants were in the randomization group or the observation group

\section{Number analyzed:}

Total: 26 eyes (22 infants)

Per group: 10 eyes probed at 12-14 months; 16 eyes no treatment

Unit of analysis: eye

Intervention 2: delay of probing until age 24 months

Length of follow-up: not reported

Outcomes

Primary outcome, as defined in study reports: complete or near complete remission of symptoms and signs and a normal FDDT

Secondary outcomes, as defined in study reports: not reported

Adverse events reported: yes, "No serious infective complications occurred in this study, or in our study on children in the first year of life. However, a few cases of orbital cellulitis considered secondary to lacrimal sac infection are cited in the literature and the parents should be advised of this."

Intervals at which outcomes assessed: probing arm assessed within 1 month of the procedure; delayed arm assessed at 15 months

$$
\text { Type of study: published }
$$

Funding sources: "The work was supported by a grant from the Speed-Pollock Trust."

Disclosures of interest: none reported

Study period: not reported

Reported subgroup analyses: no

Trial registration: not reported

\section{Risk of bias}

\begin{tabular}{lll}
\hline Bias & Authors' judgement & Support for judgement \\
\hline $\begin{array}{ll}\text { Random sequence genera- } \\
\text { tion (selection bias) }\end{array}$ & Low risk & $\begin{array}{l}\text { "If informed consent was obtained the child was registered by telephone with } \\
\text { the study office and a trial number allocated from random number tables (sets } \\
\text { of } 1-16) . \text { Children with odd trial numbers were placed in group } 2 \text { and probed } \\
\text { between } 12 \text { and } 14 \text { months. Those with even trial numbers were placed in }\end{array}$ \\
\hline
\end{tabular}


Young 1996 (Continued)

group 3 and probing delayed until 24 months in the hope of spontaneous remission."

\begin{tabular}{lll}
\hline $\begin{array}{l}\text { Allocation concealment } \\
\text { (selection bias) }\end{array}$ & Unclear risk & Not reported. \\
\hline $\begin{array}{l}\text { Masking of participants } \\
\begin{array}{l}\text { and personnel (perfor- } \\
\text { mance bias) }\end{array}\end{array}$ & Low risk & $\begin{array}{l}\text { The allocation is not masked to participants and personnel due to the nature } \\
\text { of the treatments in this study, but we do not suspect performance bias for the } \\
\text { primary outcome. }\end{array}$ \\
\hline
\end{tabular}

\begin{tabular}{|c|c|c|}
\hline $\begin{array}{l}\text { Masking of outcome as- } \\
\text { sessment (detection bias) }\end{array}$ & Unclear risk & Not reported. \\
\hline $\begin{array}{l}\text { Incomplete outcome data } \\
\text { (attrition bias) } \\
\text { All outcomes }\end{array}$ & High risk & $\begin{array}{l}\text { Primary outcome, 'cure,' was measured at different times for different study } \\
\text { arms. For those randomized to the immediate probing group, "cure from prob- } \\
\text { ing was defined as resolution of signs and symptoms within } 1 \text { month of the } \\
\text { procedure." For participants randomized to the observation/deferred probing } \\
\text { group, follow-up was when the participant was } 15 \text { months of age, to determine } \\
\text { if spontaneous resolution had occurred. } \\
\text { "For three infants not brought back to the clinic, telephone follow-up was pos- } \\
\text { sible." "6 were excluded because of incomplete data or lost follow-up," the } \\
\text { reasons of exclusion or loss to follow-up are unclear and the report does not } \\
\text { note to which intervention group these participants had been randomized. }\end{array}$ \\
\hline $\begin{array}{l}\text { Selective reporting (re- } \\
\text { porting bias) }\end{array}$ & Unclear risk & Study protocol is not available \\
\hline Other bias & Low risk & None \\
\hline
\end{tabular}

NLDO: nasolacrimal duct obstruction.

Characteristics of excluded studies [ordered by study ID]

\begin{tabular}{ll}
\hline Study & Reason for exclusion \\
\hline Al-Faky 2015 & Study compared different surgical procedures. \\
\hline Chaabouni 1993 & Not an RCT \\
\hline Ekinciler 1994 & Not an RCT \\
\hline Hernandez 1967 & Not an RCT \\
\hline Ishikawa 1990 & Not an RCT \\
\hline Robb 1985 & Not an RCT \\
\hline
\end{tabular}

$\mathrm{RCT}$ : randomized controlled trial.

DATA AND ANALYSES 
Comparison 1. Immediate probing vs observation/deferred probing

\begin{tabular}{lllll}
\hline Outcome or subgroup title & No. of studies & $\begin{array}{l}\text { No. of partici- } \\
\text { pants }\end{array}$ & Statistical method & Effect size \\
\hline $\begin{array}{l}1 \text { Treatment success at 18 months of } \\
\text { age }\end{array}$ & 1 & & $\begin{array}{l}\text { Risk Ratio (M-H, Fixed, 95\% } \\
\text { Cl) }\end{array}$ & Subtotals only \\
\hline $\begin{array}{l}1.1 \text { Eyes of participants with unilateral } \\
\text { NLDO }\end{array}$ & 1 & 146 & $\begin{array}{l}\text { Risk Ratio (M-H, Fixed, 95\% } \\
\text { Cl) }\end{array}$ & 1.13 [0.99, 1.28] \\
\hline $\begin{array}{l}1.2 \text { Eyes of participants with bilateral } \\
\text { NLDO }\end{array}$ & 1 & 108 & Risk Ratio (M-H, Fixed, 95\% & $0.86[0.70,1.06]$ \\
\hline
\end{tabular}

Analysis 1.1. Comparison 1 Immediate probing vs observation/ deferred probing, Outcome 1 Treatment success at 18 months of age.

\begin{tabular}{|c|c|c|c|c|c|}
\hline Study or subgroup & $\begin{array}{c}\text { Immediate } \\
\text { probing } \\
\mathbf{n} / \mathbf{N}\end{array}$ & $\begin{array}{c}\text { Deferred } \\
\text { probing } \\
n / N\end{array}$ & $\begin{array}{c}\text { Risk Ratio } \\
\text { M-H, Fixed, 95\% CI }\end{array}$ & Weight & $\begin{array}{c}\text { Risk Ratio } \\
\text { M-H, Fixed, } 95 \% \mathrm{Cl}\end{array}$ \\
\hline \multicolumn{6}{|c|}{ 1.1.1 Eyes of participants with unilateral NLDO } \\
\hline PEDIG 2012 & $69 / 75$ & $58 / 71$ & - & $100 \%$ & $1.13[0.99,1.28]$ \\
\hline Subtotal $(95 \% \mathrm{Cl})$ & 75 & 71 & & $100 \%$ & $1.13[0.99,1.28]$ \\
\hline \multicolumn{6}{|c|}{ Total events: 69 (Immediate probing), 58 (Deferred probing) } \\
\hline \multicolumn{6}{|c|}{ Test for overall effect: $Z=1.81(P=0.07)$} \\
\hline \multicolumn{6}{|c|}{ 1.1.2 Eyes of participants with bilateral NLDO } \\
\hline PEDIG 2012 & $41 / 58$ & $41 / 50$ & - & $100 \%$ & $0.86[0.7,1.06]$ \\
\hline Subtotal $(95 \% \mathrm{Cl})$ & 58 & 50 & & $100 \%$ & $0.86[0.7,1.06]$ \\
\hline \multicolumn{6}{|c|}{ Total events: 41 (Immediate probing), 41 (Deferred probing) } \\
\hline \multicolumn{6}{|c|}{ Test for overall effect: $Z=1.38(P=0.17)$} \\
\hline \multicolumn{6}{|c|}{ Test for subgroup differences: $\mathrm{Chi}^{2}=4.51, \mathrm{df}=1(\mathrm{P}=0.03), \mathrm{I}^{2}=77.8 \%$} \\
\hline
\end{tabular}

\section{APPENDICES}

\section{Appendix 1. CENTRAL search strategy}

\#1 MeSH descriptor: [Lacrimal Apparatus Diseases] explode all trees

\#2 MeSH descriptor: [Lacrimal Apparatus] explode all trees

\#3 ((obstruct ${ }^{\star}$ or block ${ }^{\star}$ or drain ${ }^{\star}$ or flow* or occlus ${ }^{\star}$ or occlude ${ }^{\star}$ ) near/4 (nasolacrimal or lacrimal or tear duct $\left.{ }^{\star}\right)$ )

\#4 NLDO or NLO

\#5 epiphor*

\#6 \#1 or \#2 or \#3 or \#4 or \#5

\#7 MeSH descriptor: [Dacryocystorhinostomy] explode all trees

\#8 (dacryocystorhinostom* or dacryocystostom*)

\#9 DCR

$\# 10$ prob $^{\star}$

$\# 11 \# 7$ or \#8 or \#9 or \#10

\#12 MeSH descriptor: [Infant] explode all trees

\#13 MeSH descriptor: [Child, Preschool] explode all trees 
\#14 MeSH descriptor: [Pediatrics] explode all trees

$\# 15$ newborn ${ }^{\star}$ or neonate or infant $^{\star}$ or child ${ }^{\star}$ or paediatric ${ }^{\star}$ or pediatric ${ }^{\star}$ or congenital ${ }^{\star}$

\#16 nurser* or kindergarten* or preschool* or pre school*

$\# 17 \# 12$ or \#13 or \#14 or \#15 or \#16

$\# 18 \# 6$ and \#11 and \#17

\section{Appendix 2. MEDLINE Ovid search strategy}

1. exp lacrimal apparatus disease/

2. exp lacrimal apparatus/

3. ((obstruct\$ or block\$ or drain\$ or flow\$ or occlus\$ or occlude\$) adj4 (nasolacrimal or lacrimal or tear duct\$)).tw.

4. (NLDO or NLO).tw.

5. epiphor\$.tw.

6. or/1-5

7. exp dacryocystorhinostomy/

8. (dacryocystorhinostom\$ or dacryocystostom\$).tw.

9. DCR.tw.

10. prob\$.tw.

11. or/7-10

12. exp infant/

13. exp child, preschool/

14. exp pediatrics/

15. congenital.fs.

16. (newborn $\$$ or neonate $\$$ or infant $\$$ or child $\$$ or paediatric $\$$ or pediatric $\$$ or congenital\$).tw.

17. (nurser $\$$ or kindergarten $\$$ or preschool\$ or pre school\$).tw.

18. or $/ 12-17$

19. 6 and 11 and 18

The search filter for trials at the beginning of the MEDLINE strategy is from the published paper by Glanville 2006.

\section{Appendix 3. EMBASE.com search strategy}

\#1 'lacrimal gland disease'/exp

\#2 'lacrimal apparatus'/exp

\#3 ((obstruct* OR block* OR drain* OR flow* OR occlus* OR occlude*) NEAR/4 (nasolacrimal OR lacrimal OR 'tear duct' OR 'tear ducts')):ab,ti \#4 nldo:ab,ti OR nlo:ab,ti

\#5 epiphor*:ab,ti

\#6 \#1 OR \#2 OR \#3 OR \#4 OR \#5

$\# 7$ 'dacryocystorhinostomy'/exp

\#8 dacryocystorhinostom*:ab,ti OR dacryocystostom*:ab,ti

\#9 dcr:ab,ti

$\# 10$ prob*$^{\star}: a b, \mathrm{ti}$

\#11 \#7 OR \#8 OR \#9 OR \#10

$\# 12$ 'newborn'/exp

\#13 'infant'/exp

\#14 'child'/exp

$\# 15$ 'pediatrics'/exp

\#16 newborn*:ab,ti OR neonate*:ab,ti OR infant*:ab,ti OR child* :ab,ti OR paediatric*:ab,ti OR pediatric*:ab,ti OR congenital*:ab,ti

\#17 nurser*:ab,ti OR kindergarten*:ab,ti OR preschool*:ab,ti OR (pre NEXT/1 school*):ab,ti

\#18 \#12 OR \#13 OR \#14 OR \#15 OR \#16 OR \#17

\#19 \#6 AND \#11 AND \#18

\section{Appendix 4. PubMed search strategy}

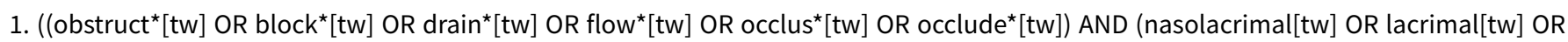
tear duct $\left.\left.{ }^{\star}[\mathrm{tw}]\right)\right)$ NOT Medline[sb]

2. (NLDO[tw] OR NLO[tw]) NOT Medline[sb]

3. epiphor ${ }^{\star}[\mathrm{tw}]$ NOT Medline[sb]

4. \#1 OR \#2 OR \#3

5. (dacryocystorhinostom* ${ }^{\star}[\mathrm{tw}]$ OR dacryocystostom* ${ }^{\star}$ tw]) NOT Medline[sb]

6. DCR[tw] NOT Medline[sb]

7. (probe ${ }^{\star}[\mathrm{tw}]$ OR probing* $\left.[\mathrm{tw}]\right)$ NOT Medline[sb]

8. \#5 OR \#6 OR \#7

9. (newborn ${ }^{\star}[\mathrm{tw}]$ OR neonate $[\mathrm{tw}]$ OR infant ${ }^{\star}[\mathrm{tw}]$ OR child*${ }^{\star}[\mathrm{tw}]$ OR paediatric ${ }^{\star}[\mathrm{tw}]$ OR pediatric ${ }^{\star}[\mathrm{tw}]$ OR congenital $\left.{ }^{\star}[\mathrm{tw}]\right)$ NOT Medline $[\mathrm{sb}]$

Probing for congenital nasolacrimal duct obstruction (Review) 
10. (nurser ${ }^{\star}[\mathrm{tw}]$ OR kindergarten ${ }^{\star}[\mathrm{tw}]$ OR preschool ${ }^{\star}[\mathrm{tw}]$ OR pre school*) NOT Medline[sb]

11. \#9 OR \#10

12. \#4 AND \#8 AND \#11

\section{Appendix 5. LILACS search strategy}

(nasolacrimal or lacrimal or epiphor\$ or Lagrimal or MH:A09.371.463\$ or MH:A10.336.422\$) and (newborn\$ or "Recién Nacido" or "RecémNascido" or MH:M01.060.703.520\$ or neonate\$ or infant\$ or Lactante or Lactente or MH:M01.060.703\$ or child\$ or Niño or Criança or MH:M01.060.406\$ or MH:SP4.011.127.413.654\$ or paediatric\$ or pediatric\$ or nurser $\$$ or kindergarten\$ or preschool\$ or pre school\$ or Preescolar or Pré-Escolar) and (Dacryocystorhinostom $\$$ or Dacriocistorrinostom $\$$ or Dacriocistorinostom\$ or Dacryocystostom\$ or DCR or $\mathrm{MH}: \mathrm{E} 04.540 .255$ or MH:E04.579.255\$ or prob\$ or sondeo or sondagem)

\section{Appendix 6. metaRegister of Controlled Trials search strategy}

(lacrimal OR nasolacrimal OR epiphor\% OR tear duct\%) AND (dacryocystorhinostom\% OR probe OR probing OR probed)

\section{Appendix 7. Clinicaltrials.gov search strategy}

(lacrimal OR nasolacrimal OR epiphora OR tear duct) AND (dacryocystorhinostomy OR probe OR probing OR probed)

\section{Appendix 8. ICTRP search strategy}

lacrimal AND dacryocystorhinostomy OR lacrimal AND probe OR lacrimal AND probing OR lacrimal AND probed OR nasolacrimal AND dacryocystorhinostomy OR nasolacrimal AND probe OR nasolacrimal AND probing OR nasolacrimal AND probed OR epiphora AND dacryocystorhinostomy OR epiphora AND probe OR epiphora AND probing OR epiphora AND probed OR tear duct AND dacryocystorhinostomy OR tear duct AND probe OR tear duct AND probing OR tear duct AND probed

\section{CONTRIBUTIONSOF AUTHORS}

Conceiving the review: DL, CP

Designing the review: DL, CP

Coordinating the review: $\mathrm{DL}, \mathrm{CP}, \mathrm{XH}, \mathrm{EC}$

Data collection for the review:

- Designing search strategies: LR, CP

- Undertaking searches: LR

- Screening search results: DL, CP

- Organizing retrieval of papers: XH

- Screening retrieved papers against inclusion criteria: $\mathrm{XH}, \mathrm{EC}$

- Appraising quality of papers: $\mathrm{XH}, \mathrm{EC}$

- Extracting data from papers: $\mathrm{XH}, \mathrm{EC}$

Data management for the review

- Entering data into RevMan: XH, EC

- Analysis of data: XH, EC, CP, DL

Interpretation of data

- Providing a methodological perspective: $\mathrm{XH}, \mathrm{EC}$

- Providing a clinical perspective: $\mathrm{DL}, \mathrm{CP}$

Writing the review: $\mathrm{EC}, \mathrm{XH}, \mathrm{CP}, \mathrm{DL}$

Updating the review: CP, DL, Cochrane Eyes and Vision US Project

\section{DECLARATIONS OF INTEREST}

None known. 


\section{SOURCES OF SUPPORT}

\section{Internal sources}

- Department of Ophthalmology at the University of Missouri Health Care in Columbia and the Missouri Ophthalmologic Society (MOSEPS), USA.

Internal funding for Carisa Petris.

\section{External sources}

- National Eye Institute, National Institutes of Health, USA.

Methodological support provided by the Cochrane Eyes and Vision Group US Project, which is funded by grant 1 U01 EY020522

- National Institute for Health Research (NIHR), UK.

- Richard Wormald, Co-ordinating Editor for Cochrane Eyes and Vision (CEV) acknowledges financial support for his CEV research sessions from the Department of Health through the award made by the National Institute for Health Research to Moorfields Eye Hospital NHS Foundation Trust and UCL Institute of Ophthalmology for a Specialist Biomedical Research Centre for Ophthalmology.

- This review was supported by the National Institute for Health Research, via Cochrane Infrastructure funding to the CEV UK editorial base.

The views and opinions expressed therein are those of the authors and do not necessarily reflect those of the Systematic Reviews Programme, NIHR, NHS or the Department of Health.

\section{DIFFERENCES BETWEEN PROTOCOLANDREVIEW}

Clarified the secondary outcome "proportion of participants with treatment success at time points beyond six months after probing" to be "proportion of participants with treatment success at time points other than six months after probing" so that time points earlier than six months could be included. This outcome is referred to as "treatment success at other time points."

\section{INDEX TERMS}

\section{Medical Subject Headings (MeSH)}

Age Factors; Anti-Bacterial Agents [therapeutic use]; Cost-Benefit Analysis; Dacryocystorhinostomy [adverse effects] [economics] [instrumentation] [ ${ }^{\star}$ methods]; Lacrimal Duct Obstruction [ ${ }^{\star}$ congenital] [ ${ }^{\star}$ therapy]; Massage; Randomized Controlled Trials as Topic; Remission, Spontaneous; Reoperation [statistics \& numerical data]; Surgical Instruments; Time Factors; Watchful Waiting

\section{MeSH check words}

Humans; Infant 